\title{
PREVALENCIA DE EVENTOS ADVERSOS EN CENTROS DE HEMODIÁLISIS
}

\section{PREVALENCE OF ADVERSE EVENTS IN HEMODIALYSIS CENTERS}

\author{
LORETO ORTEGa LoBos * \\ NALDY FeBRÉ ${ }^{* *}$
}

\begin{abstract}
RESUMEN
Los centros de diálisis son unidades complejas que predisponen la ocurrencia de eventos adversos (EA); éstos son un problema grave en todo el mundo, uno de cada 10 pacientes sufre un evento adverso durante la hospitalización. Objetivo: Determinar los eventos adversos que ocurren con mayor frecuencia en centros de hemodiálisis crónica. Material y método: Estudio descriptivo, cuantitativo, retrospectivo, realizado en tres centros de hemodiálisis del Sur de Chile. Resultados: En 241 pacientes evaluados hubo una prevalencia de un 75\% de eventos adversos; la hipotensión fue la más frecuente con un 36,04\% del total de EA. Hubo diferencias entre las prevalencias de cada centro (p-valor $<0,05$ ). Conclusiones: Durante un mes de tratamiento, 7 de cada 10 pacientes en hemodiálisis presentan eventos adversos; si bien la prevalencia en esta población puede ser similar, pareciera haber diferencias asociadas a las prácticas clínicas de cada centro, advirtiendo esfuerzos urgentes en seguridad del paciente.
\end{abstract}

Palabras clave: Enfermería en nefrología; eventos adversos; hemodiálisis; seguridad del paciente.

\begin{abstract}
Dialysis centers are complex units prone to the occurrence of adverse events (AE), which are a serious problem throughout world, as it is known that 1 in 10 patients suffers an adverse event during hospitalization. Objective: To determine which adverse events happen most frequently in chronic hemodialysis centers. Material and methods: Descriptive, quantitative, retrospective study, conducted in three hemodialysis centers, in the South of Chile. Results: There was a $75 \%$ prevalence of adverse events among the 241 patients evaluated; hypotension was the most frequent with $36.04 \%$ prevalence. There were differences between the prevalence rates in each center ( $\mathrm{p}$-value $<0.05)$. Conclusions: During one month of treatment, 7 out of 10 patients undergoing hemodialysis present an adverse event; although the prevalence of adverse events in this population may be similar, there seems to be differences associated with the clinical practices of each center, noting that urgent efforts are needed regarding safety patient.
\end{abstract}

Key words: Nephrology nursing; adverse event; hemodialysis; patient safety.

Fecha recepción: 11/03/15 Fecha aceptación: 15/05/18

\footnotetext{
* Enfermera, Magíster en gestión de seguridad de pacientes y calidad de Atención. Enfermera jefe del Centro de Diagnóstico y Tratamiento Hospital Militar de Santiago, Chile. Email: loretega@gmail.com

"Enfermera, Doctora en Ciencias de la Salud. Profesor Titular, Directora de línea de investigación de calidad y seguridad del paciente, Universidad Andrés Bello, Facultad de Enfermería, Chile. Email: naldy.febre@unab.cl. Autor de correspondencia.
} 


\section{INTRODUCCIÓN}

Diversos estudios han demostrado que los sistemas sanitarios se enfrentan a riesgos en sus procesos asistenciales, que podrían dar origen a eventos adversos ${ }^{(1-6)}$. Un evento adverso (EA) es un incidente durante la atención que produce daño en el paciente, y que no tiene relación con su enfermedad; el daño puede ser físico, social o psicológico $^{(7,8)}$.

La OMS ${ }^{(7)}$ estandarizó la información relacionada con la seguridad del paciente, con el propósito de formular una nomenclatura y clasificación de los eventos adversos que facilite la comparación y aprendizaje local, regional, nacional e internacional; desarrollando la clasificación internacional de seguridad del paciente, la cual se compone por 13 clases superiores de eventos adversos organizadas de la siguiente manera: 1) administración clínica; 2) proceso/procedimiento clínico; 3) documentación; 4) infección asociada a la atención de salud; 5) medicación/líquidos para administración endovenosa; 6) sangre/ productos sanguíneos; 7) nutrición; 8) oxígeno/ gases y vapores; 9) dispositivos/equipos médicos; 10) comportamiento; 11) accidentes de los pacientes; 12) infraestructura/locales/instalaciones; 13) recursos/gestión de la organización.

Los eventos adversos aumentan la morbimortalidad de los pacientes y constituyen un gran problema de salud pública mundial, es por esto que la seguridad de atención debe ser considerada como un componente fundamental de los sistemas sanitarios ${ }^{(9)}$.

La seguridad del paciente se define como la reducción del riesgo de daño innecesario asociado a la atención de salud ${ }^{(7,8)}$, y a pesar de ser conocida desde la época hipocrática, con la ética médica y el principio de no maleficencia, su transformación en un cuerpo de conocimiento específico es reciente, y se ha desarrollado con mayor fuerza en el ámbito hospitalario, existiendo en el área ambulatoria prácticas y entornos sanitarios igualmente riesgosos, tal cual las unidades de hemodiálisis, en donde una compleja conjugación de factores predispone a mayores oportunidades de errores en los procesos de atención.

En Chile existen 21.233 pacientes en hemodiálisis crónica ${ }^{(9)}$; esta población ha aumentado más de 30 veces en los últimos 25 años ${ }^{(10)}$, con cambios considerables en su perfil demográfico, destacando entre ellos la edad de los pacientes que resulta cada vez mayor, en donde 1 de cada 3 pacientes supera los 70 años ${ }^{(9)}$. Estos usuarios, a su vez, son portadores de múltiples morbilidades, incluyendo entre ellas una alta prevalencia de Diabetes Mellitus, con el deterioro adicional que aporta esta patología. La carga de enfermedad hace que la polifarmacia sea omnipresente, y se estima que cada paciente en terapia dialítica recibe en promedio de 7 a 10 medicamentos al día ${ }^{(11,}$ ${ }^{12)}$; el $64,5 \%$ de los pacientes recibe a lo menos un hipotensor ${ }^{(9)}$, que en combinación con los trastornos hidroelectrolíticos favorecen la hipotensión y las caídas posteriores al procedimiento. Se ha descrito que la población en hemodiálisis, que supera los 65 años, tiene mayor riesgo de caídas que la población general ${ }^{(13-15)}$.

Los errores de medicación también son frecuentes en este tipo de pacientes, en especial por dosis equívocas de anticoagulantes u omisión de otros medicamentos prescritos ${ }^{(16,17)}$. En esta línea, el paciente renal posee una condición inmunológica disfuncional ${ }^{(18,19)}$ que en presencia de factores contribuyentes como dispositivos invasivos, resistencia antimicrobiana ${ }^{(20)}$ y poca adhesión a las medidas de prevención ${ }^{(21)}$ hacen de las unidades de diálisis ambientes favorables para el desarrollo de infecciones asociadas a la práctica sanitaria.

La calidad del agua y el reprocesamiento del circuito utilizado en el procedimiento son áreas claves tanto en la prevención de infecciones como en otro tipo de eventos. Se han establecido recomendaciones internacionales para evitar la ocurrencia de eventos adversos asociados al reúso de la membrana, sin embargo, se han notificado errores de identificación de circuitos, con la conexión del paciente equivocado ${ }^{(22)}$.

En nuestro país, el gasto en salud ha crecido a una tasa real anual de 9\%, duplicándose en el periodo comprendido entre 1990-2012 (1,6 a $3,5 \%)^{(23)}$. Desde 2006 este gasto ha aumentado 
asociado a la implementación del régimen de Garantías Explícitas en Salud (GES). El manejo de la enfermedad renal crónica es parte de las Garantías Explícitas en Salud, con un gasto de $22 \%$ del presupuesto GES al año 2008, equivalente a 170 mil millones de pesos; siendo el problema de salud con el mayor costo esperado por beneficiario en el año 2016, además es la patología de mayor variación en el costo (periodo 2012-2015), con un incremento de 10,4\% (23, 24). Este incremento en el gasto se explica por el ingreso de pacientes a terapia de hemodiálisis, que ha aumentado de manera sostenida, desde 10.693 personas el año 2005 a 19.071 personas al año 2015, representando un aumento de 78,5\% en los últimos 10 años ${ }^{(24)}$.

Actualmente en Chile existen 249 centros/ hospitales que realizan terapia dialítica trisemanal, el $81,5 \%$ de ellos privados ${ }^{(21)}$. De acuerdo a la regulación existente en Chile, las prestaciones GES solo pueden ser otorgadas por prestadores acreditados, siendo, en el caso de los centros de diálisis, exigible esta acreditación de calidad a partir de julio de $2018^{(25)}$. A marzo de 2017 el $6,4 \%$ de éstos se encuentran acreditados con el estándar para Centros de Diálisis de la Superintendencia de Salud ${ }^{(25)}$. El estándar de acreditación de Centros de Diálisis considera, en el ámbito Gestión Clínica (componentes GCL-2, GCL3), la vigilancia de eventos adversos asociados a la atención y las infecciones asociadas al acceso vascular (este indicador es el único que tiene definida su vigilancia desde el año 1998). Actualmente las necesidades de información en relación a los eventos adversos ocurridos en unidades de hemodiálisis en nuestro país sobrepasan las proporcionadas por el actual sistema de vigilancia basado en reportes voluntarios del personal de estas unidades, constatándose una metodología pasiva, la cual presenta baja sensibilidad (10 y $30 \%$ ), realizada mediante método no sistemático, que impide obtener tasas de estos eventos a nivel nacional ${ }^{(26)}$. La inexistencia en nuestro país y a nivel internacional de un sistema de vigilancia epidemiológico con metodología activa que se aplique sistemáticamente es imprescindible para mejorar el conocimiento en relación con la seguridad del paciente sometido a hemodiálisis crónica, por medio de la aproximación a la magnitud, trascendencia e impacto de los eventos adversos (EA) y al análisis de las características de los pacientes y de la asistencia, que se asocian a la aparición de EA evitables.

Los cuidados enfermeros durante el tratamiento de hemodiálisis son muy importantes para que la asistencia que reciba el paciente sea de calidad. Enfermería entiende que los efectos variados sobre la salud, tales como los factores socioeconómicos, culturales, sociales, sumado a la combinación de un procedimiento que exige interacción humana y alta tecnología en un grupo de pacientes con una patología crónica, permite que el paciente este expuesto a numerosos riesgos, que en su mayoría son evitables ${ }^{(27)}$.

En las unidades de hemodiálisis, el equipo de enfermería exhibe el mayor involucramiento en los procesos de asistencia sanitaria, lo que permite que estos profesionales, por su mayor cercanía con los pacientes, tengan un rol relevante en la seguridad de éstos y en la calidad asistencial, pero también sean agentes claves para reducir los resultados de los eventos adversos ${ }^{(28)}$. En Chile, la legislación ha definido el rol social de las enfermeras, asignándole, entre otras funciones, la "gestión del cuidado", en especial con la Norma Administrativa No 19, que aporta antecedentes que otorgan las bases orgánicas funcionales de las Unidades de Gestión del Cuidado ${ }^{(4)}$.

Es por lo anteriormente expuesto que el objetivo del presente estudio fue identificar los eventos adversos que ocurren con mayor frecuencia en las unidades de hemodiálisis ambulatorias, así como también reconocer algunos factores de riesgo que permitan en la práctica diaria orientar los esfuerzos en seguridad, y planificar intervención sobre la base de información local.

\section{MATERIAL Y MÉTODO}

Se efectuó un estudio cuantitativo descriptivo, retrospectivo, a través de una prevalencia de eventos adversos. El estudio se realizó en tres cen- 
tros de diálisis del Sur de Chile, donde se realizó muestreo por conveniencia; como criterio de inclusión se consideró a los pacientes que cumplían con un tiempo de permanencia en el centro igual o superior a 30 días, y que recibían hemodiálisis en esquema trisemanal. No se incluyeron los pacientes hospitalizados durante el periodo en estudio, y aquellos con acceso vascular transitorio, trasladados de otros centros de diálisis.

En el periodo comprendido entre el 1 al 31 de julio del año 2013 fueron evaluados un total de 251 pacientes en los tres centros analizados. Para la recogida de datos se utilizaron dos instrumentos: el primero para obtener datos epidemiológicos y detectar eventos adversos, el segundo para clasificar el evento, clasificar el daño y el momento del evento. Ambos instrumentos fueron elaborados por los autores y validados previamente por 3 expertos de distintas instituciones, vinculados al área calidad y seguridad del paciente y nefrología, los que fueron consultados acerca de su opinión experta sobre ambos instrumentos creados por los investigadores. Para esto se realizaron dos rondas de consulta, con el objetivo de comprobar que los diferentes ítems y dimensiones de las primeras versiones de los instrumentos permitieran medir los constructos o dominios originales, además de evaluar si el lenguaje empleado se adapta a la población en la que se aplicaría. Las observaciones y los cambios propuestos por los integrantes del grupo de expertos fueron revisadas por los investigadores del estudio para posteriormente incluirlos y finalizar con una segunda versión consensuada del instrumento. Para la validación en campo, las dos versiones finales fueron aplicadas a un grupo de 10 pacientes en tratamiento de hemodiálisis trisemanal, previo consentimiento informado, en un centro de hemodiálisis diferente a los analizados; en esta etapa se observó el tiempo utilizado para completar el instrumento, preguntas o datos que les generen duda o confusión, los cuales fueron modificados, hasta contar con las versiones finales de los instrumentos.

Los datos se obtuvieron de los registros médicos y de enfermería, de todas las sesiones de hemodiálisis efectuadas en el periodo de estudio.
La recolección de datos estuvo a cargo de una enfermera por cada centro participante, previa capacitación en la aplicación de instrumentos de recolección, conceptos claves según la taxonomía de la OMS ${ }^{(12)}$, y finalmente taller de presentación del estudio y reconocimiento de eventos. La asignación del centro se hizo en forma aleatoria, para evitar que las enfermeras recogieran información de su propia unidad.

Para la definición de los eventos adversos se utilizó el marco conceptual de la clasificación internacional para la seguridad del paciente, fueron registrados el tiempo de ocurrencia del evento adverso (antes, durante o después del procedimiento de hemodiálisis) y se determinó el grado de daño resultado del EA (ninguno, leve, moderado, grave y muerte) ${ }^{(12)}$.

Se realizó análisis descriptivo, con porcentajes en las variables cualitativas, y medidas de centralización y dispersión. Las diferencias estadísticas se determinaron por prueba de Chi cuadrado, con un nivel de significancia $p$-valor $\leq$ a 0,05 , fue utilizado el software para análisis estadístico SPSS.

El estudio fue aprobado por el Comité de Ética de la Facultad de Medicina de la Universidad Mayor, así como también por las autoridades de los centros participantes. Fueron respetados los requisitos éticos de Ezequiel Emanuel.

\section{RESULTADOS}

Se obtuvo una muestra de 251 pacientes, se excluyeron 10 pacientes por haber presentado hospitalizaciones durante el periodo en estudio, la proporción de pérdida fue de un 4,0\%. Se incluyeron para el análisis un total de 241 pacientes, distribuidos en tres centros de diálisis, donde el centro 2 aportó con el 40,2\% del total.

La edad media de los pacientes fue de 60,2 años, con una desviación estándar de 13,8 años y una mediana de 62 años (amplitud intercuartil: 16 años). Al comparar los pacientes por edad, sexo y centro, se encontró que tanto hombres como mujeres presentaron una edad media simi- 
lar, a excepción del centro 3, en donde las mujeres tuvieron una edad media superior a los hombres: 61,34 años y 55,3 años, respectivamente.

Respecto a la antigüedad en hemodiálisis, la media del grupo en estudio fue de 4,6 años, y una mediana de 3 años (amplitud intercuartil: 6 años). Del total de los pacientes, el 34,44\% eran hipertensos, mientras que el $11 \%$ eran diabéticos; y un $28,22 \%$ padecían ambas patologías. En el centro 3, sólo el 3,80\% estaban libres de Hipertensión Arterial (HTA) y/o Diabetes Mellitus
(DM). En cuanto a los accesos vasculares, el 15\% de los pacientes se dializaban a través de un catéter central para hemodiálisis.

De los 241 pacientes estudiados, 181 presentaron eventos adversos, lo que corresponde a una prevalencia del 75\%. El total de eventos adversos detectados fue de 677. El 72,38\% de los pacientes prevalentes presentó entre uno y cuatro EA, el 21\% entre cinco y nueve, y finalmente 6,63\% entre diez y trece EA (Gráfico 1).

Gráfico 1. Distribución de pacientes según frecuencia de eventos adversos detectados en los tres centros de diálisis evaluados $(n=241)$.

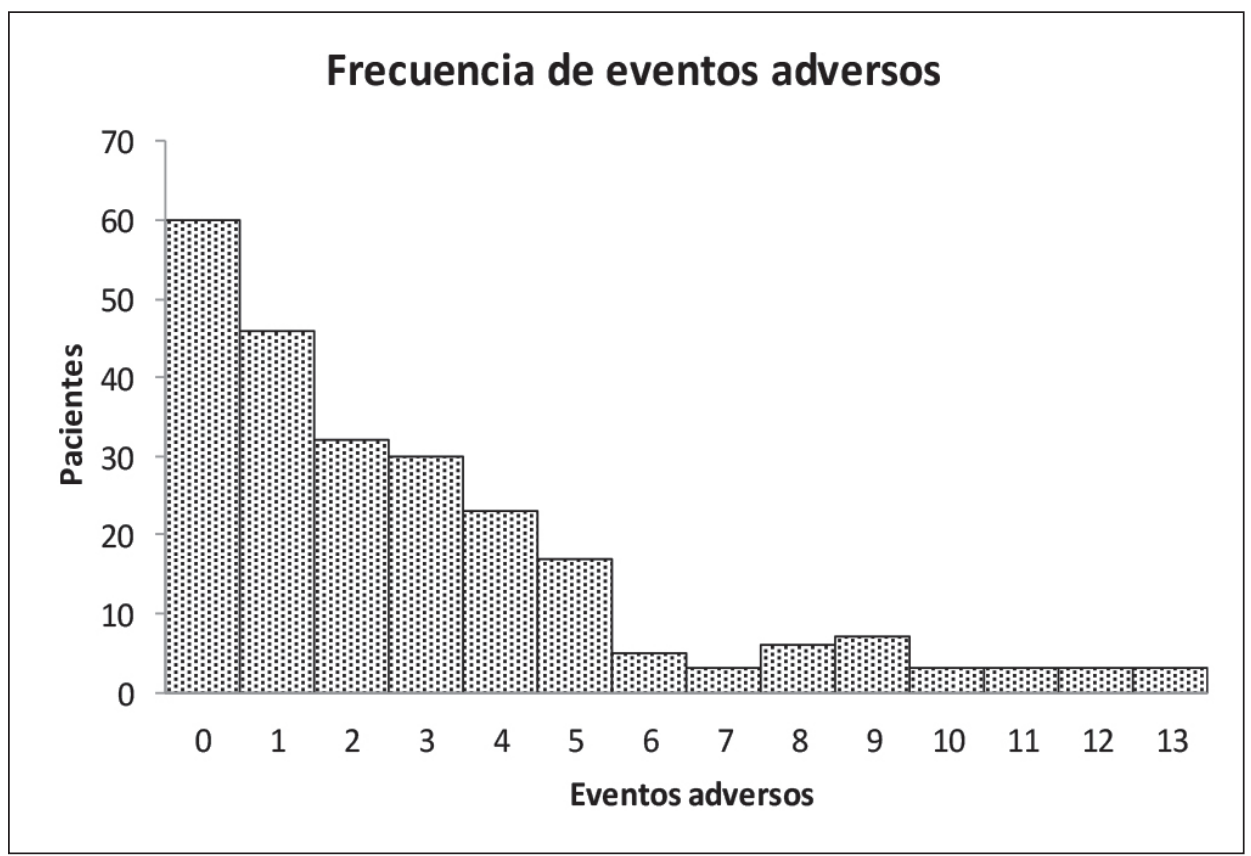

Del total de sesiones de hemodiálisis evaluadas un $27,7 \%$ presentaron eventos adversos (mediana de 21,34\% y desviación estándar de $21,98 \%)$. Hubo diferencias en las prevalencias de cada centro, siendo mayor en el centro 3, luego en el centro 1 y centro 2, respectivamente (Gráfico 2); se encontró relación estadísticamente significativa entre el centro y la ocurrencia de EA (Chi cuadrado igual a 1,96; p-valor de 0,005 en la prueba de asociación (Tabla 1).

$\mathrm{Al}$ analizar la prevalencia de EA, por edad y sexo, se encontraron mayores prevalencias en algunos grupos, destacando el grupo entre 42 a 49 años del centro 1, con una prevalencia de 57,9\%. Las mayores prevalencias se observaron en los grupos de menor edad. Respecto al género de los pacientes, la mediana es ligeramente mayor en las mujeres; con un Odds ratio (OR) de EA de 1,18 (IC: 0,65-2.11). Finalmente, al realizar las pruebas de asociación, no se encontró significancia estadística en ninguno de los grupos, por edad o por sexo. 
Gráfico 2. Prevalencia media (IC: 95\%) de eventos adversos por centro de diálisis evaluado (n=181).

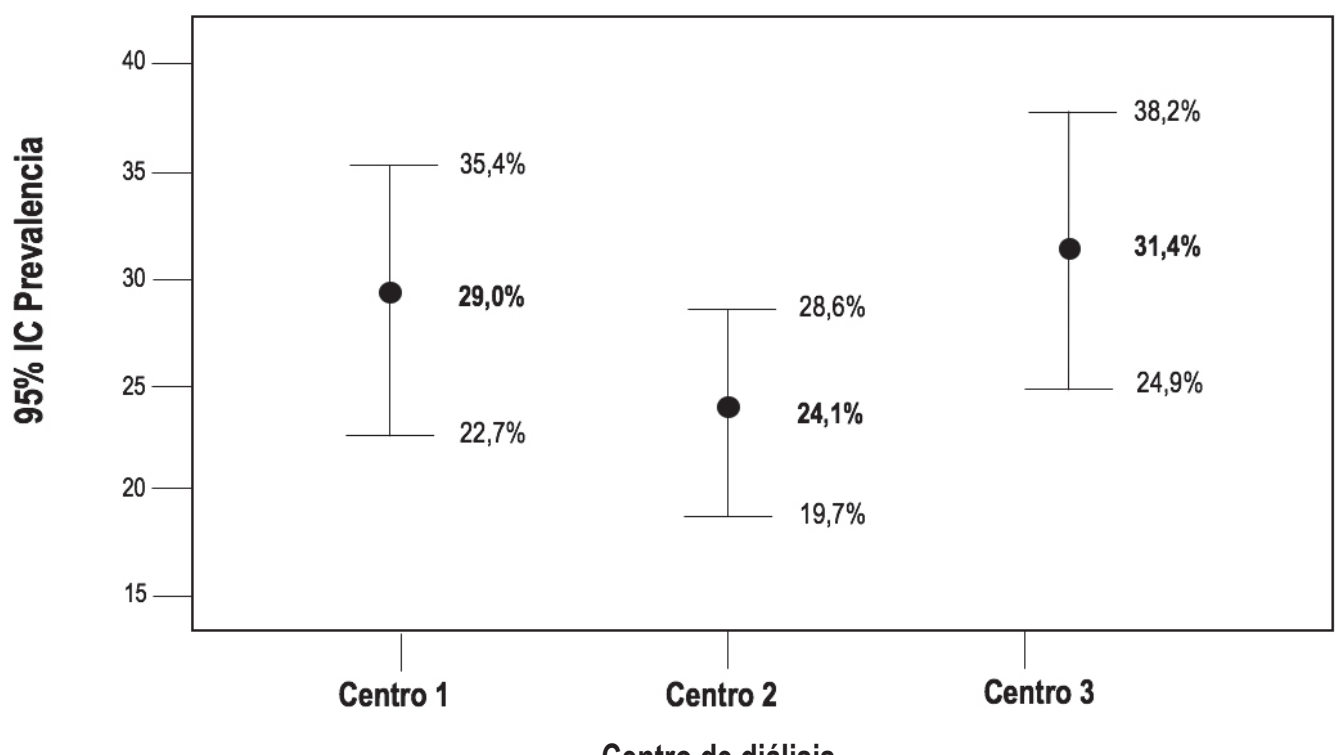

Centro de diálisis

Tabla 1. Proporción de pacientes según presencia de eventos adversos detectados por centro de diálisis evaluado $(\mathrm{n}=241)$.

\begin{tabular}{lrrrrrrrrr}
\hline & \multicolumn{2}{c}{ Centro 1 } & \multicolumn{2}{c}{ Centro 2 } & \multicolumn{2}{c}{ Centro 3 } & \multicolumn{2}{c}{ Total } & \\
\cline { 1 - 7 } & $\mathrm{n}$ & \multicolumn{1}{c}{$\%$} & $\mathrm{n}$ & \multicolumn{1}{c}{$\%$} & \multicolumn{1}{c}{$\mathrm{n}$} & \multicolumn{1}{c}{$\%$} & $\mathrm{n}$ & $\%$ & p-valor $\left(\chi^{2}\right)$ \\
\hline Con EA & 56 & 23,24 & 75 & 31,12 & 50 & 20,75 & 181 & 75,10 & \multirow{2}{*}{0,0055} \\
Sin EA & 9 & 3,73 & 22 & 9,13 & 29 & 12,03 & 60 & 24,90 & \\
\hline Total & 65 & 26,97 & 97 & 40,25 & 79 & 32,78 & 241 & 100,00 & 0,0055 \\
\hline
\end{tabular}

La prevalencia de eventos adversos según presencia de hipertensión, diabetes, o ambas, se muestra en la Tabla 2; en el centro 2 se aprecia una prevalencia mayor en hipertensos (Chi cuadrado 2,3; p-valor $<0,005$ ).

Fueron detectados 6 tipos de EA (Tabla 3). La hipotensión fue el EA más frecuente con un 36\% del total. La suma de la hipotensión, desconexión anticipada $(24,96 \%)$ y calambres $(18,76 \%)$ representa el $80 \%$. Cada centro presentó diferencias de distribución, el centro 1 tuvo una mayor proporción de desconexión anticipada, mientras los centros 1 y 2 hipotensión, seguida de calambres; los errores de medicación fueron más frecuentes en el segundo centro; el esquema de diálisis semanal incompleto fue similar en los centros 2 y 3. Finalmente los hematomas tuvieron mayor frecuencia en el centro 3.

Todos los EA detectados están asociados al procedimiento y ocurrieron durante la sesión de hemodiálisis. El 15,82\% de los eventos no generaron daño evidente; el 84,11\% desencadenó daño leve; no se describieron eventos con daños moderados, graves, ni muerte. 
Tabla 2. Prevalencia media de pacientes con eventos adversos por centro de diálisis evaluado, según presencia de patologías $(n=181)$.

\begin{tabular}{|c|c|c|c|c|c|c|c|c|c|}
\hline \multirow{2}{*}{ Centro } & & \multicolumn{2}{|c|}{ HTA } & \multicolumn{2}{|c|}{$\mathrm{DM}$} & \multirow[b]{2}{*}{ p-valor $\left(\chi^{2}\right)$} & \multicolumn{2}{|c|}{$\mathrm{HTA}+\mathrm{DM}$} & \multirow[b]{2}{*}{$\mathrm{p}$-valor $\left(\chi^{2}\right)$} \\
\hline & & No & $\mathrm{Si}$ & No & $\mathrm{Si}$ & & No & $\mathrm{Si}$ & \\
\hline \multirow{2}{*}{1} & $\mathrm{n}$ & 22 & 34 & 36 & 20 & \multirow{2}{*}{0,103} & 17 & 15 & \multirow{2}{*}{0,672} \\
\hline & $\%$ & 22,30 & 21,40 & 21,40 & 21,40 & & 23,10 & 21,40 & \\
\hline \multirow{2}{*}{2} & $\mathrm{n}$ & 45 & 30 & 47 & 28 & \multirow{2}{*}{0,005} & 34 & 17 & \multirow{2}{*}{0,293} \\
\hline & $\%$ & 15,40 & 19,00 & 15,40 & 16,00 & & 15,40 & 16,70 & \\
\hline \multirow{2}{*}{3} & $\mathrm{n}$ & 6 & 44 & 27 & 23 & \multirow{2}{*}{0,279} & 2 & 19 & \multirow{2}{*}{0,109} \\
\hline & $\%$ & 17,90 & 28,60 & 25,00 & 28,60 & & 46,40 & 28,60 & \\
\hline \multirow{2}{*}{ Total } & & 73 & 108 & 110 & 71 & \multirow{2}{*}{0,07} & 53 & 51 & \multirow{2}{*}{0,403} \\
\hline & & 20,00 & 21,40 & 21,40 & 21,40 & & 21,40 & 23,10 & \\
\hline
\end{tabular}

HTA=Hipertensión arterial; DM=Diabetes Mellitus.

Tabla 3. Distribución según tipo de eventos adversos detectados por centro de diálisis evaluado ( $\mathrm{n}=$ 677).

\begin{tabular}{lrrrrrrrr}
\hline \multirow{2}{*}{ Eventos Adversos } & \multicolumn{2}{c}{ Centro 1 } & \multicolumn{2}{c}{ Centro 2 } & \multicolumn{2}{c}{ Centro 3 } & \multicolumn{2}{c}{ Total } \\
\cline { 2 - 10 } & \multicolumn{1}{c}{$\mathrm{n}$} & \multicolumn{1}{c}{$\%$} & \multicolumn{1}{c}{$\mathrm{n}$} & \multicolumn{1}{c}{$\%$} & \multicolumn{1}{c}{$\mathrm{n}$} & \multicolumn{1}{c}{$\%$} & \multicolumn{1}{c}{$\mathrm{n}$} & $\%$ \\
\hline Hipotensión & 35 & 15,22 & 100 & 42,37 & 109 & 51,66 & 244 & 36,04 \\
Desconexión anticipada & 146 & 63,48 & 6 & 2,54 & 17 & 8,06 & 169 & 24,96 \\
Calambres & 22 & 9,57 & 61 & 25,85 & 44 & 20,85 & 127 & 18,76 \\
Errores de medicación & 10 & 4,35 & 42 & 17,80 & 11 & 5,21 & 63 & 9,31 \\
Esquema semanal de diálisis incompleto & 8 & 3,48 & 21 & 8,90 & 19 & 9,00 & 48 & 7,09 \\
Hematoma & 9 & 3,91 & 6 & 2,54 & 11 & 5,21 & 26 & 3,84 \\
\hline Total & 230 & 100 & 236 & 100 & 211 & 100 & 677 & 100 \\
\hline
\end{tabular}

\section{DISCUSIÓN}

Los eventos adversos son un problema de salud que también afecta a los centros de diálisis ambulatoria; según este estudio, 7 de cada 10 pacientes en hemodiálisis crónica presentan eventos adversos durante un mes de tratamiento. Esta elevada prevalencia (75\%) concuerda con la encontrada por el National Healthcare Safety Network (NHSN) en los Estados Unidos, quienes comunican que de un total de 6.005 centros de hemodiálisis para pacientes ambulatorios para el año 2014 existieron 160.971 eventos adversos asociados al procedimiento de hemodiálisis ambulatoria ${ }^{(29)}$.

Esta institución (NHSN), mediante evaluación retrospectiva de reportes de EA, comunica la ocurrencia de 24.092 eventos adversos en el periodo 2007-2011, identificándolos como: necesidad de hospitalización, inicio de terapia antibiótica e infección del acceso vascular ${ }^{(30)}$. Matarán Robles et al., mediante vigilancia pasiva, reportaron la ocurrencia de 141,96 eventos adversos por cada 1.000 procedimientos en un estudio realizado en un periodo de un año calendario ${ }^{(31)}$. 
En cuanto a los EA detectados, en el presente estudio la hipotensión fue el más frecuente con un $36,5 \%$; en coincidencia con el estudio de incidencia de Sousa et al. ${ }^{(32)}$, en donde representó el 55,4\%; el mismo estudio describe en segundo lugar un inadecuado tratamiento dialítico con un 20,1\%; la segunda frecuencia en prevalencia en la presente investigación es el evento adverso de desconexión anticipada con un $24,96 \%$, que también podría calificar como tratamiento inadecuado.

Los errores de medicación con 9,31\% ocuparon, en los tres centros evaluados, el cuarto lugar en frecuencia a diferencia de lo descrito por Holley, que los posiciona en primer lugar ${ }^{(17)}$. Por otro lado, a pesar de que el 15\% de los pacientes se dializaba a través de un catéter venoso central, no hubo eventos relacionados a este tipo de acceso, como lo reportado por Sousa et al. ${ }^{(32)}$, esta diferencia puede estar definida por el diseño metodológico, poco comparable.

Existen estudios que han relacionado la edad de los pacientes en $\mathrm{HD}$, con un mayor riesgo de caídas ${ }^{(13,14)}$; y han sido descritas como eventos adversos por Holley ${ }^{(17)}$, Matarán Robles et al. ${ }^{(31)}$ y Sousa et al. ${ }^{(32)}$; en esta investigación no se detectaron caídas, pero no debemos olvidar que los resultados pueden ser poco exactos, dado que la información se obtuvo por la revisión de fichas clínicas, pudiendo existir un sesgo por registro. Si bien el impacto en cuanto al grado de daño descrito no es excesivo, es esencial reconocer los errores en las prácticas clínicas, y de especial interés la desconexión anticipada, que sin mayor análisis se puede catalogar como un evento adverso prevenible, independiente de su gravedad, por estar sujeto a una acción voluntaria del personal de enfermería responsable de los cuidados.

La inasistencia a hemodiálisis no ha sido descrita como evento adverso en otros estudios, quizás porque el daño no se evidencia en forma inmediata, pero dosis insuficientes de hemodiálisis aumentan la morbimortalidad de los pacientes, y éste es un efecto perjudicial, al considerar que tanto la desconexión anticipada como la inasistencia disminuyen la dosis de hemodiálisis. Siguiendo las orientaciones de la OMS ${ }^{(33)}$, se debe incorporar al paciente como parte del proceso de atención, en este caso en particular, para prevenir la inasistencia, y asegurar la dosis prescrita; sin embargo, pueden existir factores que valen la pena mencionar, como la extrema ruralidad, que en ocasiones no permite que el paciente asista en forma regular.

La diferencia en las prevalencias de eventos adversos por centro fue estadísticamente significativa ( $p$-valor 0,005 ), siendo mayor la prevalencia media del centro 3 (31,5\%; IC: 24,9-38,2), atribuible quizás a la gran carga de enfermedades crónicas asociadas en los pacientes, ya que, en este centro, sólo el 3\% de los pacientes estaban libres de Hipertensión Arterial y Diabetes Mellitus. Se debe reconocer bajo esta premisa, que cada centro tiene una realidad propia, y que toda estrategia de intervención debiera estar sujeta a las características de cada institución.

Los profesionales enfermeros, que trabajan en unidades de hemodiálisis ambulatorias poseen una responsabilidad legal, ética y social con la gestión del cuidado, lo que los obliga a asumir un compromiso y una responsabilidad basada en asegurar la continuidad y calidad de los cuidados entregados.

Éste estudio permite un primer acercamiento a la epidemiología de los eventos adversos que ocurren con mayor frecuencia en pacientes en hemodiálisis crónica trisemanal ambulatoria en nuestro país.

Los resultados encontrados desprenden una necesidad urgente de generar información adicional, en especial complementar estudios de prevalencias, con incidencias y seguimientos prospectivos para reconocer los factores de riesgo en la inmediatez de lo sucedido, asimismo poder dimensionar el impacto real, a través del seguimiento de los pacientes, ya que el modelo utilizado en esta investigación podría suponer una validez cuestionable por el sesgo de registro. Además, resulta conveniente unificar criterios, definiciones y sistemas de notificación, que funcionen dentro de un contexto de clima de calidad y seguridad, con una cultura que aprenda de los errores y que no tema reconocerlos.

En ese escenario es posible pensar en la ins- 
talación de sistemas de vigilancia epidemiológica activa a cargo de un profesional enfermero, y gestión de riesgos como herramientas efectivas de prevención, en beneficio de la seguridad y bienestar del paciente.

Se destaca que los pacientes en hemodiálisis crónica reconocen su vulnerabilidad, el 50\% de ellos piensa que un evento adverso se producirá durante su tratamiento ${ }^{(34)}$. La vulnerabilidad ha sido expresada como un elemento central para la declaración universal de bioética y derechos del hombre, considerando que los pacientes con ERC en hemodiálisis requieren atención de salud segura, que garantice el respeto a la dignidad humana e información oportuna ${ }^{(34)}$.

\section{CONCLUSIONES}

Los resultados del presente estudio permiten concluir que en los tres centros analizados se encontró una tasa de prevalencia de eventos adversos igual a $75 \%$ (181 / 241 pacientes), con un total de 677 eventos adversos vigilados, siendo que el $84,11 \%$ presentó daño para el paciente clasificado como leve; se destaca que en los tres centros evaluados existieron diferencias en las prevalencias de los eventos adversos.

No se encontró diferencia por edad o sexo del paciente; los eventos adversos detectados fueron: hipotensión (36,0\%); desconexión anticipada $(24,9 \%)$; calambres $(18,7 \%)$; errores de medicación (9,3\%); esquema semanal incompleto $(7,0 \%)$ y hematomas $(3,8 \%)$.

Se debe considerar que el presente estudio descriptivo-retrospectivo no controló la asignación del paciente, sino que ésta se efectúo de acuerdo a la práctica clínica habitual, permitiendo una aproximación retrospectiva al evento de interés (eventos adversos durante proceso de hemodiálisis); por el tipo de estudio existe una limitada comparación entre los grupos y sesgo de registros, lo que impide establecer causalidad. Sin embargo, los investigadores entienden que la presente investigación constituye el primer paso para futuras investigaciones en esta importante área de enfermería.

Estos datos permiten comprender que la frecuencia de eventos adversos en las unidades de hemodiálisis ambulatoria estudiadas es elevada (7 de cada 10 pacientes presentaron un eventos adverso), situación que hace necesario la formación de capacidades de enfermería para comprender los factores que explican esta prevalencia; como profesionales de enfermería dedicados a la gestión del cuidado debemos realizar esfuerzos colaborativos de los distintos niveles para el desarrollo conjunto de un sistema de vigilancia activo que permita desarrollar indicadores orientados a evaluar la gestión del cuidado, permitiendo estructurar acciones de gestión (programas de intervenciones locales), dirigidas a generar impactos positivos en la salud de estos pacientes vulnerables.

El segundo punto a considerar es la necesidad de la integración de grupos de investigación en el que participen enfermeras clínicas y académicos investigadores de las diferentes escuelas y facultades de Enfermería, con el objetivo de potenciar una línea de investigación en vigilancia epidemiológica activa e impacto de diferentes intervenciones sobre la seguridad del paciente en hemodiálisis, que entregue evidencia científica de alta calidad a los administradores y formuladores de políticas públicas en salud a nivel nacional, obteniendo un mayor valor del cuidado enfermero en unidades de hemodiálisis ambulatorias.

\section{REFERENCIAS}

1. Leape LL, Brennan TA, Laird N, Lawthers AG, Localio AR, Barnes BA, et al. The nature of adverse events in hospitalized patients. Results of the Harvard Medical Practice Study II. N Engl J Med. 1991; 324(6): 377-84.

2. Wilson RM, Runciman WB, Gibberd RW, Harrison BT, Newby L, Hamilton JD. The Quality in Australian Health Care Study. Med J Aust. 1995; 163(9): 458-71.

3. Institute of Medicine (US), Committee on Quality of Health Care in America, Kohn LT, 
Corrigan JM, Donaldson MS. editors. To Err Is Human: Building a Safer Health System. Washington (DC): National Academies Press (US); 2000.

4. Milos P, Bórquez B, Larraín S. La "Gestión del Cuidado" en la Legislación Chilena: Interpretación y Alcance. Cienc. enferm. 2010; XVI(1): 17-29.

5. Aranaz-Andrés JM, Aibar-Remón C, LimónRamírez R, Amarilla A, Restrepo FR, Urroz $\mathrm{O}$, et al. Prevalence of adverse events in the hospitals of five Latin American countries: results of the 'Iberoamerican Study of Adverse Events' (IBEAS). BMJ Qual Saf. 2011; 20(12): 1043-51.

6. Aranaz-Andrés JM, Aibar-Remón C, Vitaller-Burillo J, Ruiz-López P. Estudio Nacional sobre los Efectos Adversos ligados a la Hospitalización. ENEAS 2005. Madrid: Ministerio de Sanidad y Consumo España; 2006. 169 p.

7. Organización Mundial de la Salud. Seguridad del Paciente [Internet]. Ginebra: Organización Mundial de la Salud; 2004 Oct [citado 06 mayo 2018]. 68 p. Disponible en: http://www.who.int/patientsafety/es/

8. Organización Mundial de la Salud. Marco Conceptual de la clasificación internacional para la seguridad del paciente. Versión 1.1 [Internet]. Ginebra: Organización Mundial de la Salud; 2009 [citado 06 enero 2013]. 160 p. Disponible en: http://www.who.int/about/ copyright/es/index.html

9. Sociedad Chilena de Nefrología, Registro de diálisis III. XXXVII Cuenta de hemodiálisis crónica (HDC) en Chile (al 31 de agosto de 2016) [Internet]. Valparaíso: Sociedad Chilena de Nefrología; 2017 [citado 10 enero 2018]. Disponible en: http://www.nefro.cl/ test/web2/articulo.php?id=623

10. Flores JC, Alvo M, Borja H, Morales J, Vega J, Zúñiga C, et al. Enfermedad renal crónica: Clasificación, identificación, manejo y complicaciones. Rev Med Chil. 2009; 137: 137-77.

11. Bray BD, Boyd J, Daly C, Doyle A, Donaldson $\mathrm{K}$, Fox JG, et al. How safe is renal replacement therapy? A national study of mortality and adverse events contributing to the death of renal replacement therapy recipients. Nephrol Dial Transplant. 2014; 29: 681-687.

12. Katzir Z, Boaz M, Backshi I, Cernes R, Barnea Z, Biro A. Medication and apprehension compliance among dialysis patients-a comprehensive guidance attitude. Nephron Clin Pract. 2010; 114(2): c151-7.

13. Albuquerque SE, Cavalcante RS, Ponce D, Fortaleza CMCB. Epidemiology of infections, healthcare, patients, hemodialysis and the southeast of Brazil. Braz J Infect Dis. 2014; 18(3): 327-330.

14. Olsha O, J Hijazi, Goldin I, Shemesh D. Vascular access in hemodialysis patients older than 80 years. J Vasc Surg. 2015; 6(1): 177183.

15. Hakim RM, Collin AJ. Reducing avoidable re-hospitalization in ESRD: a shared accountability. J Am Soc Nephrol 2014; 25(9): 1891 1893.

16. Badawy DA, Mowafi HS, Al-Mousa HH. Surveillance of dialysis events: 12-Month experience at five outpatient adult hemodialysis centers in Kuwait. J Infect Public Health. 2014; 7(5): 386-391.

17. Holley JL. A descriptive report of errors and adverse events in chronic hemodialysis units. Nephrol News Issues. 2006; 20(12): 57-63.

18. Lok CE, Sontrop JM, Faratro R, Chan CT, Zimmerman DL. Frequent hemodialysis fistula infectious complications. Nephron Extra. 2014; 4(3): 159-167.

19. Kliger AS. Maintaining Safety in the Dialysis Facility. Clin J Am Soc Nephrol. 2015; 10(4): 688-95.

20. Al-Jaishi AA, Liu AR, Lok CE, Zhang JC, Moist LM. Complications of arteriovenous fistula: a systematic review: J Am Soc Nephrol. 2016; 28(6): 1839-50.

21. Ulrich B, Kear T. Patient safety culture in nephrology nurse practice settings: initial findings. Nephrol Nurs J. 2014; 41(5): 459475.

22. Centers for Disease Control and Prevention (CDC). Recommendations for Preventing Transmission of Infections Among Chronic 
Hemodialysis Patients [Internet]. Atlanta: CDC, Morbidity and Mortality Weekly Report, Recommendations and Reports; 2001 Apr [citado 11 abril 2018]; 43 p. Disponible en: https://www.cdc.gov/mmwr/preview/ mmwrhtml/rr5005a1.htm

23. Benavides P, Castro R, Jones I. Sistema Público de Salud. Situación Actual y Proyecciones Fiscales 2013-2050 [Internet]. 2013 [citado 11 abr 2017]; 108 p. Disponible en: http:// www.dipres.gob.cl/598/articles-117505_ doc_pdf.pdf

24. Sociedad Chilena de Nefrología, XXV Cuenta de hemodiálisis crónica (HDC) en Chile (al 31 de agosto de 2006) [Internet]. Lugar: Sociedad Chilena de Nefrología; 2007 [citado 11 abr 2017]. Disponible en: http://www. nefro.cl/hd2005.pdf

25. Superintendencia de Salud de Chile. Registros de entidades reguladas por la Superintendencia de Salud [Internet]. Santiago, Chile: Ministerio de Salud; 2012 [citado 26 mar 2017]. Disponible en: http://www.supersalud.gob.cl/acreditacion/673/w3-article-9006. html

26. Superintendencia de Salud de Chile. Manual del estándar general de acreditación para centros de diálisis [Internet]. Santiago, Chile: Ministerio de Salud; 2014 [citado 27 mar 2017]. Disponible en: http://www.supersalud.gob.cl/observatorio/671/articles-4530_ manual_CD_pdf.pdf

27. Sniehotta FF, Araújo-Soares V, Brown J, Kelly MP, Michie S, West R, et al. Complex systems and individual-level approaches to population health: a false dichotomy? The Lancet Public Health. 2017; 2(9): e396-e397.

28. Wakefield BJ. Facing up to the reality of missed care. BMJ Qual Saf. 2014; 23(2): 92-4.

29. Nguyen DB, Shugart A, Lines C, Shah AB, Edwards J, Pollock D, et al. National Healthcare Safety Network (NHSN) Dialysis Event Surveillance Report for 2014. Clin J Am Soc Nephrol. 2017; 12(7): 1139-46.

30. Patel PR, Shugart A, Mbaeyi C, Goding Sauer A, Melville A, Nguyen DB, et al. Dialysis Event Surveillance Report: National Healthcare Safety Network data summary, January 2007 through April 2011. Am J Infect Control. 2016; 44(8): 944-7.

31. Matarán Robles EM, Aguilar García R, Muñoz Becerra M. Incidencia y tipo de efectos adversos durante el procedimiento de hemodiálisis. Enferm Nefrol. 2013; 16(1): 36-40.

32. Sousa MRG, Silva AEBC, Bezerra ALQ, Freitas JS, Miasso AI. Adverse events in hemodialysis: reports of nursing professionals. Rev Esc Enferm USP. 2013; 47(1): 75-82.

33. Organización Mundial de la Salud. Alianza Mundial para la Seguridad del Paciente. La Investigación en Seguridad del Paciente. Mayor conocimiento para una atención más segura [Internet]. Ginebra: OMS; 2008 [citado 01 mar 2014]. Disponible en: http://www. who.int/patientsafety/information_centre/ documents/ps_research_brochure_es.pdf

34. Waldow VR. Cuidado humano: la vulnerabilidad del ser enfermo y su dimensión de trascendencia. Index Enferm. 2014; 23(4): 234-8. 